\title{
Flexibilización cuantitativa: características e impacto en las economías de los Estados Unidos y Colombia
}

\author{
Alberto Parra Barrios \\ Universidad Militar Nueva Granada \\ albertoparra9@yahoo.com
}

DOI: https://doi.org/10.21158/01208160.n82.2017.1646

Fecha de recepción: 20 de mayo de 2016

Fecha de aprobación: 27 de julio de 2016

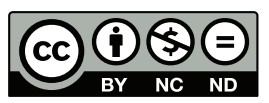

Cómo citar este artículo/ To reference this article/ Comment citer cet article/ Para citar este artigo:

Parra, A. (2017). Flexibilización cuantitativa: características e impacto en las economías de los Estados Unidos y Colombia. Revista EAN, 82, pp 13-35. https://doi.org/10.21158/01208160.n82.2017.1646

\section{Resumen}

Este trabajo tiene como propósito describir la política monetaria implementada por la Federal Reserve System entre 2008 y 2015, teniendo en cuenta sus objetivos, la teoría económica que la sustenta y el impacto en los principales indicadores de las economías estadounidense y colombiana. Para el desarrollo metodológico, se tuvo en cuenta la investigación aplicada de tipo documental y la aplicación de un modelo de mínimos cuadrados ordinarios que muestra el impacto del programa en indicadores de la economía colombiana, a partir del modelo de valoración de activos financieros (Capital Asset Pricing Mode/- CAPM). Los resultados muestran el efecto del programa en los agregados monetarios, la tasa de desempleo y los activos financieros de renta fija en los Estados Unidos y el impacto en el índice COLCAP, la tasa representativa del mercado (TRM), el precio del crudo de Texas (WTI) y la inversión extranjera directa (IED) en Colombia entre 2010 y 2015.

\section{Palabras clave}

Flexibilización cuantitativa, Reserva Federal, canales de transmisión, GSE-MBS.

\footnotetext{
1 Administrador de Empresas de la Pontificia Universidad Javeriana. Especialista en Finanzas por la Universidad de Los Andes. Magíster en Finanzas y Mercados Financieros por la Universidad San Pablo CEU en España. MBA con énfasis en Finanzas por la Universidad Sergio Arboleda. Maestría en Economía por la Pontificia Universidad Javeriana. Docente de Finanzas en la Facultad de Ciencias Económicas y Administrativas de la Universidad Militar Nueva Granada. ORCID: http://orcid.org/0000-0003-1173-3077
} 


\title{
Quantitative easing: features and impact on the economies of USA and Colombia
}

\begin{abstract}
This paper describes the monetary policy implemented by The Federal Reserve System during 2008- 2015, taking into account its objectives, its economic theory and the impact of main indicators in USA and Colombia. For its methodological development, a former documented research and the application of an ordinary, square minimal model, which shows the program impact on the indicators of Colombian economy, were taken into account, based on a Capital Asset Pricing Model (CAPM). Its outcomes show the program impact on money supplies, the representative market exchange rate and the fixed rate of financial assets in USA as well as its direct impact on COLCAP, the representative market exchange rate, the oil price in Texas (WTI) and direct foreign investment (IED) in Colombia during 2008- 2015.
\end{abstract}

Key words. Quantitative eaisng, Federal Reserve, transmission channels, GSE- MBS.

\section{Assouplissement quantitatif: caractéristiques et impact asur les économies colombiennes et étasuniennes}

Resumé. Ce travail a pour objectif de décrire la politique monétaire mise en œuvre par la Réserve Fédérale américaine entre 2008 et 2015 en tenant compte de ses objectifs, de la théorie économique qui la sous-tend et de l'impact sur les principaux indicateurs des économies américaines et colombiennes. Notre méthodologique prend en compte la recherche appliquée de type documentaire et l'application d'un modèle de minimums carrés ordinaires montrant l'impact du programme en terme d'indicateurs sur l'économie colombienne à partir du modèle d'évaluation d'actifs financiers (Asset Pricing Capital Model - CAPM). Les résultats mettent en évidence les effets du programme sur les agrégats monétaires, sur le taux de chômage et les actifs financiers de rente fixe aux États-Unis, l'impact sur l'indice COLCAP, le taux de change peso/dollar (TRM), le prix du brut du Texas (WTI) et l'investissement direct étranger (IED) en Colombie entre 2010 et 2015.

Mots clefs. Assouplissement quantitatif, Réserve Fédérale, canaux de transmission, GSE-MBS.

\section{Flexibilização quantitativa: características e impacto nas economias dos Estados Unidos e da Colômbia}

Resumo. Este trabalho tem como propósito descrever a política monetária implementada pela Federal Reserve System entre 2008 e 2015, considerando seus objetivos, a teoria econômica que a sustenta e seus impactos nos principais indicadores das economias estadunidense e colombiana. Para o desenvolvimento metodológico, considerou-se a pesquisa aplicada de tipo documental e a aplicação de um modelo de mínimos quadrados ordinários que mostra o impacto do programa em indicadores da economia colombiana, a partir do modelo de valorização de ativos financeiros (Capital Asset Pricing Model - CAPM). Os resultados mostram o efeito do programa nos agregados monetários, a taxa de desemprego e os ativos financeiros de renda fixa nos Estados Unidos e o índice COLCAP, a taxa representativa do mercado (TRM), o preço do petróleo do Texas (WTI) e o investimento estrangeiro direta (IED) na Colômbia entre 2010 e 2015.

Palabras chave. Flexibilização quantitativa, Reserva Federal, canais de transmissão, GSE-MBS. 


\section{Introducción}

a crisis que se gestó en los Estados Unidos -entre 2007 y 2008 llevó a la quiebra a importantes entidades financieras en ese país, a inversionistas extranjeros de Europa, Japón y América Latina e impactó las principales bolsas del mundo y los activos financieros de renta fija. Ante esta difícil situación, los bancos centrales de los Estados Unidos, el Reino Unido, Japón y la zona euro comenzaron a implementar una serie de políticas monetarias no convencionales llamadas QE (quantitative easing), para estimular el crecimiento de sus economías y sacarlas de la recesión en que se encontraban (Barcelata, 2010; BBC Mundo, 2008; Stiglitz, 2010).

Los analistas económicos han considerado que la política de flexibilización cuantitativa implementada en estos países ha tenido efectos positivos y negativos. Para unos, en los Estados Unidos, no se lograron los resultados esperados en su economía, pero sí en las bolsas de valores del mundo (Costero, 2013). Los estudios realizados por Parodi (2013) demuestran que las facilidades de liquidez otorgadas por el banco de la Reserva Federal (FED, Federal Reserve System) como respuesta a la crisis financiera internacional de 2008 tuvieron importantes efectos sobre los flujos de capitales, los precios de activos -especialmente de vivienda-, el comportamiento del crédito y las tasas de interés de largo plazo en economías emergentes y en la economía de los Estados Unidos. Otros economistas investigadores como Leyva, Gómez, Valencia y Villamizar (2016) consideran que hubo efectos positivos en los activos financieros de las economías emergentes.

Para ver los efectos de QE en las economías de los Estados Unidos y de Colombia, este trabajo se inicia con la definición del concepto, la descripción de los acontecimientos previos a la aparición de la crisis, la implementación del programa y los objetivos de sus tres etapas, se comenta la teoría económica que sustenta el enfoque dado por la FED en cabeza de su presidente Ben Bernanke y se procede a analizar el impacto en los indicadores de interés de las economías de los Estados Unidos y de Colombia. Al final, se concluye que la flexibilización de la política monetaria en los Estados Unidos, ejercida de una manera agresiva a partir de 2008 por el banco de la FED, para responder a los problemas de su economía y la incertidumbre en los mercados financieros, interno y externo, trajo efectos positivos en los indicadores de la economía colombiana y en los activos financieros de su país, pero no tan satisfactorios como se esperaba en la tasa de desempleo de los Estados Unidos. 


\section{Antecedentes}

Cegún Stiglitz (2010), la gran recesión de - 2008 es la consecuencia inevitable de unas políticas que habían sido aplicadas a lo largo de los años precedentes: «Resulta obvio que esas políticas habían sido conformadas por intereses particulares —de los mercados financieros- y también de algunos economistas» (pp. 21-22). Antes de 2008, los Estados Unidos y gran parte de las economías del mundo estaban enfrentando muchos problemas, entre ellos, la adaptación al cambio climático. La globalización económica imponía cambios rápidos en las estructuras económicas de los países y se forzaba al máximo la adaptación de muchas economías. Aunque en el Foro de Davos, celebrado en 2007, se planteaba que el crecimiento económico mundial estaba en $7 \%$-cifra sin precedentes-, y se presentaban muy buenos augurios para África y América Latina.

A diferencia de lo que fueron las crisis anteriores a 2007-2008, como la del lunes negro de 1987, la burbuja de Japón de 1989 - la de Long Term Capital Management, para ese año los instrumentos financieros representativos no fueron las tradicionales acciones y los bonos, sino que fueron unos títulos más sofisticados, como las titulizaciones, entre ellos, los provenientes de deuda hipotecaria, llamados mortgage-backed securities (MBS) y los collateralized debt obligation (CDO) que podían incluir deuda hipotecaria, aun lo que se conoce como la segmentación de instrumentos que permiten fraccionar la deuda en dos partes.

En la práctica los MBS o CDO facilitaban empaquetar deuda hipotecaria de muy baja calidad crediticia por su alto riesgo de insolvencia. En consecuencia quienes los compraron en ese momento desconocían el componente interno de estos o en su defecto pudieron considerar que el paquete por su composición podía compensar pérdidas con ganancias y así obtener un resultado neto favorable a sus intereses financieros (Palomino, 2011,p71).

La titulización de estos activos logró que el capital prestado por las entidades bancarias se realizará de forma inmediata y se convirtiera en dinero líquido para estas instituciones, de manera que el plazo de maduración de la deuda se redujera rápidamente, sin emplear protecciones adicionales lo que permitía hacer crecer más las operaciones. A esta forma de operar financiera le acompañó un modelo de seguro, que daba confianza al mercado, denominado credit default swap (CDS) que como permuta financiera intercambiaba deuda del tenedor de un CDO con las compañías aseguradoras, quienes en caso de incumplimiento tenían que responder debido al pago periódico de una comisión o prima por los tenedores.

Este novedoso modelo de seguros incentivó las negociaciones de CDO en el sistema financiero estadounidense y con el tiempo fue la causa de la caída de Lehman Brothers -principal agente tomador del instrumento financiero- $y$ del rescate de la aseguradora AIG, que cubría los CDO con CDS. Cuando los MBS y CDO cayeron en impago, estos finalmente se tuvieron que financiar con incremento de la base monetaria (BM) de la FED. Posteriormente, se observó que, en el momento de enfrentar la crisis con las políticas de flexibilización cuantitativa, se distorsionó la curva de rendimiento de los instrumentos del Tesoro de los Estados 
Unidos, debido a que las tasas de interés del tramo corto de la curva de rendimiento de los bonos cayeron, dado que la variación de la BM se realizó con una activa participación de la FED, lo cual motivó el incremento del precio de dichos instrumentos y la caída de la tasa de interés.

Siguiendo a Stiglitz (2010, p. 32), este fue el esquema detonador de la crisis financiera del 2008: los bajos tipos de interés y una regulación laxa alimentaron la burbuja inmobiliaria. A medida que subían los precio de la vivienda, los propietarios obtenían más dinero con sus casas. Estos créditos con garantía hipotecaria, que en un año alcanzaron los USD 975000 millones, cifra equivalente a $7 \%$ del PIB, permitía que los prestatarios pagaran un nuevo vehículo y les quedara un remanente para su pensión. Pero estas operaciones se basaban en el supuesto de que los precios de la vivienda continuarían subiendo o no bajarían próximamente, lo cual implicaba un riesgo.
Dada esta situación, se puede decir que la economía de los Estados Unidos ya se encontraba desajustada, toda vez que una cifra importante de su PIB estaba relacionada con la vivienda en alguna de sus etapas de construcción, mampostería o equipamiento, y otra con préstamos sobre vivienda usada para financiar el consumo. Cuando explotó la burbuja, se afectó, primero, las hipotecas de alto riesgo, concedidas a individuos de bajos ingresos, y posteriormente a todos los propietarios del sector residencial que podrían tener más estabilidad.

Como se observa en la figura 1 , desde 2008, la FED se orientó al otorgamiento de crédito, con recortes progresivos de la tasa de interés de referencia o federal funds rate (FFR), hasta colocarla en el límite inferior de 0 a $0.25 \%$. Pero esta medida, dentro de la aplicación de instrumentos convencionales de la política monetaria, no fue suficiente para contrarrestar los efectos de la caída en los mercados y reactivar el crecimiento de su economía, entre 2008 y 2010. El M1 y M2 presentan un descenso importante, lo cual demuestra que no hubo liquidez con el crédito de bajo costo (Figura 1).

Figura 1. Crecimiento monetario en los Estados Unidos (2000-2012).

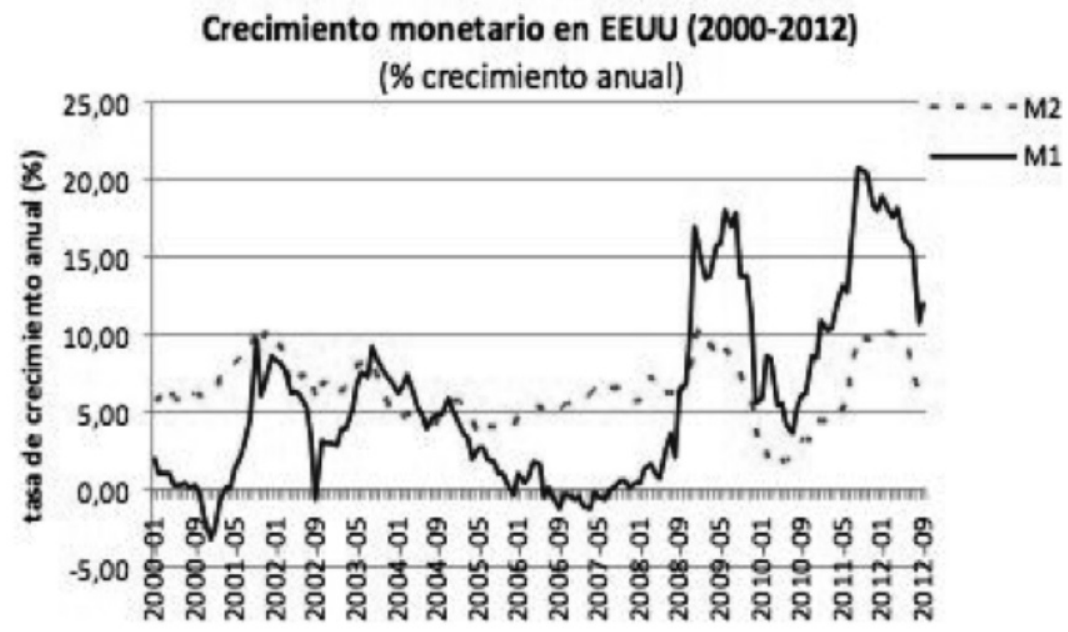

Fuente. Castañeda, (2012). 
Al presentarse «la trampa por la liquidez», según Bernanke y Blinder (2012), la FED decide implementar el conjunto de políticas no convencionales para mantener el crecimiento de la oferta monetaria, a partir de las tres etapas del programa emprendidas, las cuales se describen más adelante., con el único fin de incentivar la demanda interna.

Mientras tanto la crisis en los Estados Unidos rápidamente se volvió global, cerca de $25 \%$ de las hipotecas había salido a países de Europa y Japón (Morales, 2014).

La flexibilización cuantitativa o quantitative easing (QE) es una herramienta de política monetaria utilizada por algunos bancos centrales para aumentar la oferta de dinero que incremente el exceso de reservas del sistema bancario mediante la compra de bonos del mismo Gobierno central, para estabilizar o aumentar sus precios y con ello reducir las tasas de interés de largo plazo. Esta medida suele ser utilizada cuando los instrumentos habituales de control de la oferta de dinero no han funcionado.

El programa consiste en emitir dinero para financiar la compra de bonos y valores hipotecarios, en busca de mantener las tasas de interés de mediano y largo plazo en niveles bajos, con el propósito de estimular la demanda del crédito $y$, en consecuencia, impulsar el crecimiento económico y reducir el desempleo por el aumento del consumo y la inversión privada (Toro, 2014).

\section{Marco teórico}

En las diferentes economías mundiales, - se establecen y ejecutan políticas monetarias y políticas fiscales para alcanzar y mantener un equilibrio económico. La política monetaria es ejecutada por los bancos centrales con instrumentos de política y la política fiscal se ejecuta por el Gobierno estableciendo un sistema de recaudos para financiar el gasto público, fortalecidos con el presupuesto nacional.

\subsection{El modelo IS-LM}

La teoría económica permite explicar con el modelo Hicks-Hansen por qué la FED optó por el programa QE en sus tres etapas, para sacar de la recesión la economía de

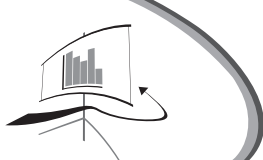
los Estados Unidos. Siguiendo a Zablotzky (2011) y a Barro, Grilli y Febrero (2004), este modelo de demanda agregada describe el equilibrio entre el ingreso nacional (Y) y los tipos de interés ( $r$ ), sobre la base de que los precios son rígidos, y permite explicar las decisiones de política monetaria emitidas por el Banco Central y las decisiones sobre política fiscal expedidas por el Gobierno.

El modelo se representa con dos curvas que se cortan; en una se tienen los mercados reales -inversión ahorro- identificados con IS $y$ en la otra los mercados monetarios -demanda de dinero oferta de dinero- identificados con LM. En el eje horizontal, se mide el ingreso nacional $(Y$ y en el eje vertical los tipos de interés $(r)$. En la práctica, los dos mercados interactúan para determinar el nivel de $Y$ que encuentra la demanda de dinero y la tasa de interés en equilibrio. A su vez, $r$ influye en el nivel de demanda de dinero que se canaliza hacia la inversión a partir de un aumento o disminución de la tasa de interés. 
Figura 2. El modelo IS-LM.

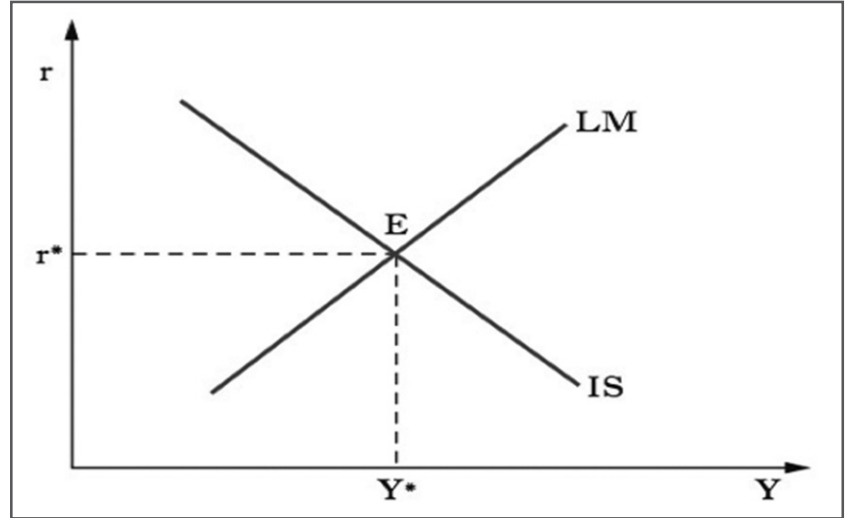

Fuente. Elaboración propia.

El modelo IS-LM, (Figura 2), muestra la curva IS, cuya pendiente es negativa a causa de que la inversión privada reacciona de manera inversa a la tasa de interés. Al bajar la tasa, aumenta la demanda agregada desplazándose la curva a la derecha, incrementando el nivel de la producción. Si aumenta la tasa de interés, el efecto es contrario: se va a producir una reducción en la inversión, con un desplazamiento de la demanda a la izquierda.

La curva LM permite analizar las posibles combinaciones de ingreso y tasa de interés, hasta obtener una tasa ajustada al llegar a alcanzar una oferta y una demanda iguales. La curva LM tiene pendiente positiva, por tanto, a un mayor nivel de ingreso hay un mayor nivel de tasa de interés, y al aumentar la oferta de dinero, se provoca bajada en la tasa del mercado de dinero y de los bonos. La oferta de dinero es exógena al modelo y es controlada por el Banco Central.

Sobre ejecución de políticas económicas, las responsabilidades están repartidas; ante las perturbaciones económicas, el equilibrio de bienes y servicios se logra con las decisiones de política fiscal que realiza el Gobierno, y en el manejo del mercado de dinero y las tasas de interés interviene el Banco Central con instrumentos de política monetaria. Ahora, como el tema tratado en este artículo es de política monetaria, siguiendo al profesor Zablotzky (2011), se pueden resaltar los efectos de la política monetaria expansiva, según el modelo HicksHansen, en la siguiente forma:

- El efecto sobre IS-LM:

- Se presenta un desplazamiento de la LM hacia la derecha por mayor oferta monetaria.

- Se presenta un aumento del ingreso de equilibrio.

- Inicialmente ocurre una disminución del tipo de interés de equilibrio.

- El efecto sobre los componentes de la demanda agregada:

- Ocurre un aumento del consumo privado al aumentar el ingreso disponible.

- El gasto público es constante.

- Hay un aumento de la inversión, dado que disminuye la tasa de interés.

- Posteriormente, aumenta la tasa de interés por una mayor demanda agregada. 


\subsection{La política monetaria expansiva con baja tasa de interés y aumento del crédito}

Una política monetaria expansiva lleva a que la oferta monetaria $\left(M^{\beta}\right)$ sea mayor a la demanda monetaria $\left(M^{d}\right)$. Cuando se reduce la tasa de interés $L M$, se desplaza $L M^{*}$ y la tasa cae de $r_{0}$ a $r_{0^{*}}$ con un nivel de ingreso

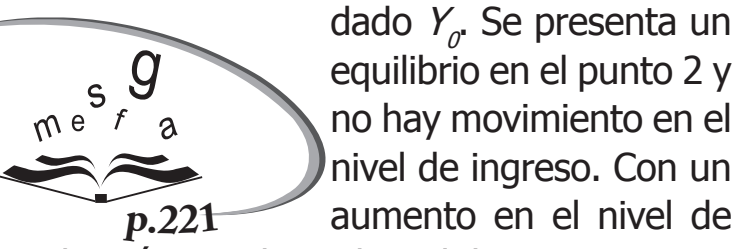
producción, se eleva el nivel de transacciones con esta influencia se genera un proceso de ajuste entre la demanda agregada y el ingreso, que lleva a una nueva tasa de interés $r_{1}$ con un nuevo equilibrio en el punto 3 que presiona el alza de la tasa de crédito (Barro, Grilli y Febrero, 2004, p. 488).

\subsection{Una política expansiva con flexibilización monetaria total (QE)}

Se parte de la existencia de un nivel de precios fijo, un aumento de la cantidad de dinero $(M)$ proveniente de la compra de bonos en el mercado abierto y una emisión de dinero a costo muy bajo o costo cero; tiene un impacto positivo en el crecimiento del PIB (Morales, 2014).

Figura 3. Efectos de una política monetaria expansiva con baja tasa de crédito.

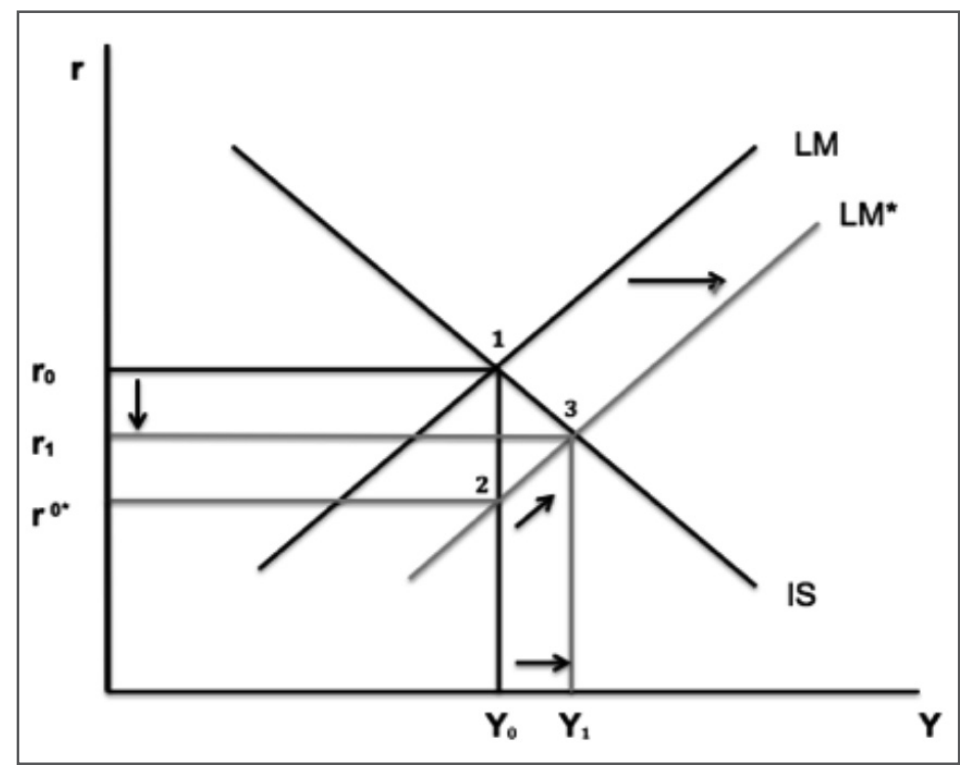

Fuente. Elaboración propia.

El impacto de la política QE presentado (Figura3) muestra un desplazamiento sucesivo de la curva $L M$, mediante una política monetaria flexible con la reducción de la tasa de interés rpor la compra de bonos del Tesoro y del sector privado; este proyecta hasta llegar a $L M^{*}$ e incrementa el ingreso hasta colocarlo en $Y n$. De esta manera, se puede afirmar que, al reducirse el tipo de interés junto con un mayor volumen de dinero en circulación, se produce un mayor crecimiento en $Y$ que el que se generaría con la sola política de reducción de la tasa de interés. 
En efecto, la política monetaria más flexible impacta la inversión con un desplazamiento a la derecha que alcanza niveles considerables de Y (Barro, Grilli y Febrero, 2004), pp. 488-489). En concordancia con lo expresado por Bernanke y Blinder (2012), los efectos positivos de QE demuestran que la financiación de la inversión en las condiciones normales, con una reducción del costo del crédito y la emisión de bonos, no es sustituto de la política monetaria expansiva QE con la cual se logra un mayor crecimiento del PIB.

\subsection{Política monetaria convencional}

Dependiendo del estado en que se encuentre la economía y de su coyuntura, el Banco Central ejecuta la política monetaria más adecuada y conveniente mediante el uso de instrumentos de política. Pretende dar estabilidad a la economía utilizando lineamientos expansivos cuando la economía se encuentra resentida o lineamientos restrictivos cuando es necesario ralentizar el crecimiento del producto interno (Morales, 2014, p. 11).

Una política monetaria expansiva busca estimular la economía. Para incrementar el crecimiento económico, el Banco Central reduce el tipo de interés de referencia (IR) o tasa repo, que es aquella a la cual presta dinero las entidades financieras que componen el sistema; simultáneamente, aumenta la BM realizando las operaciones de mercado abierto (OMA) que permiten, con la compra de valores, incrementar la base monetaria por el aumento de los depósitos bancarios (Morales, 2014; Palomino, 2013). Los resultados positivos se ven con el incremento del PIB, un incremento del IPC (índice de precios de consumo), la disminución de la tasa de desempleo y el incremento de la IED -inversión extranjera directa- (Figura 4).

Figura 4. Efectos del sucesivo descenso de LM.

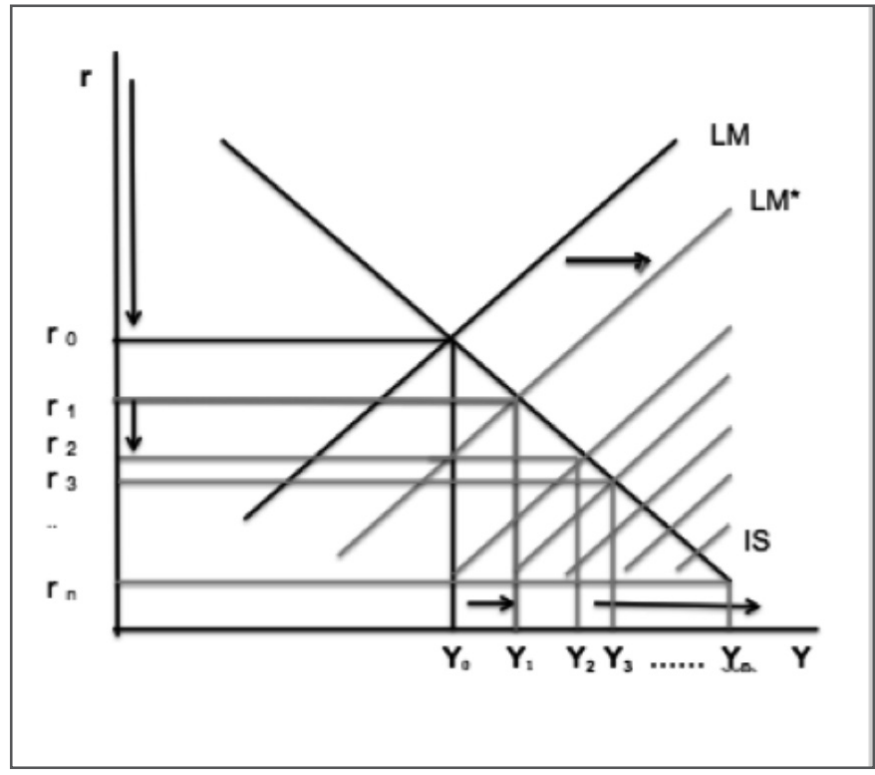

Fuente. Elaboración propia. 
Cuando se aplica una política monetaria restrictiva, se hace con el fin de controlar la inflación. Su aplicación se hace en forma contraria a las operaciones expansivas, en busca de efectos obviamente contrarios. En este orden de ideas, los tipos de interés (IR) se incrementan para limitar el crédito y el endeudamiento de las empresas y las personas, con lo cual se contrae el crecimiento. Así las cosas, al aplicar el Banco Central las OMA, vende títulos del Gobierno - TES, contrae las reservas y limita la oferta monetaria, pero sacrifica crecimiento económico (Morales, 2014, pp. 10-12).

Estas dos formas de política monetaria convencionales difieren de la flexibilización cuantitativa, por cuanto esta no busca directamente la bajada en las tasas de interés o el aumento de la base monetaria, sino la compra de bonos del Tesoro y entidades privadas que otorga una mayor liquidez al sistema para incentivar el ingreso.

\section{Canales con los cuales el Banco Central de los Estados Unidos ejecuta la expansión cuantitativa QE}

En la última semana de octubre de 2014, Ela FED puso fin a cerca de seis años de política de relajamiento cuantitativo, con un programa donde se creó dinero de la nada, con lo cual se salió de la forma convencional de emisión de efectivo para inyectar liquidez a la economía. Su objetivo fue muy claro: llegar a la economía a través de varios canales para recuperarla y sacarla adelante, después de la crisis que la llevó a la recesión. Los canales a través de los cuales se implementa la política de expansión cuantitativa se concretan en cuatro líneas (Leyva et al., 2016; Costero, 2014).

\subsection{Canal tradicional o efecto balance}

Consiste en hacer crecer 0 inflar el balance del Banco Central y, por un efecto directo con los bancos comerciales, convertir el aumento de la base monetaria en oferta monetaria. De esta manera, se disminuyen los costos de la financiación y se favorece la inversión productiva para mejorar el crecimiento del producto.

\subsection{Efecto señales}

Ocurre cuando el Banco Central transmite la idea de que se ha reducido la tasa de interés por la adopción de la política de flexibilización cuantitativa y no se incrementarán durante un tiempo considerable, en busca de la reactivación de los indicadores económicos que muestren el crecimiento del producto.

\subsection{El efecto liquidez}

Consiste en inyectar dinero al sistema con la compra de activos financieros, lo cual lleva a incrementar el precio de los títulos y a reducir la tasa de interés. La reducción del tipo ayuda a la financiación de los proyectos a bajo costo. En los Estados Unidos, el impacto de este canal ha sido fuerte, ya que el efecto se refleja vía mercado y no por el crédito bancario. 


\subsection{El canal inflación}

Se busca con la compra de activos del Tesoro recalentar la inflación con el consumo. A efectos prácticos, la QE de la FED es una línea de crédito gratuita, irrevocable y casi ilimitada en favor de la banca comercial; una rápida expansión del crédito bancario y de amplio dinero en circulación deberá hacerse con una estructura productiva bien orientada, para evitar inflación por partida doble (Costero, 2014).

\subsection{Eficacia de la política monetaria de la FED}

La política de flexibilización monetaria total, implementada en los Estados Unidos para sacar su economía de la recesión a partir de 2008, se explica en las siguientes etapas.

\subsection{La primera etapa (QE1)}

Inicia en noviembre 2008 y termina en noviembre de 2009. La FED anuncia que comprará USD 600000 millones, distribuidos USD 100000 millones en obligaciones de GSE, más USD 500000 millones en MBS3 respaldados por Fannie Mae, Freddie Mac y la Federal Homme Loan Bank las primeras compras y Fannie Mae, Freddie Mac y Ginnie Mae las segundas adquisiciones. Posteriormente, el programa se amplió en USD 300 000 millones más las adquisiciones de bonos americanos, hasta alcanzar un gran total de USD 1750000 millones, como lo anunció el presidente de la FED de San Francisco (Morales, 2014, p. 26) ${ }^{2}$.
Este primer programa inició con el sector inmobiliario y tuvo como propósito impulsar el flujo de las hipotecas, reduciendo su coste y aumentando la disponibilidad para conseguir la recuperación del sector y consecuentemente el resto de la economía, debido a que fue el más afectado por la crisis; $80 \%$ de estas compras estaban ligadas a ese sector con GSE y MBS (Morales, 2014, p. 26).

\subsection{La segunda etapa (QE2)}

Su anuncio se hizo en agosto de 2010 con un programa que inició en noviembre de ese año, con la compra de USD 600000 millones de Letras del Tesoro, para un total de USD 75000 millones mes, finalizando en junio de 2011. Esta segunda fase estaba orientada a rebajar el interés de los títulos del Tesoro de los Estados Unidos para recalentar la inflación con un aumento del consumo (Morales, 2014, p. 26).

Entre septiembre de 2011 y junio de 2012, se implementa la operación Twist con la idea de comprar USD 400000 millones de treasuries con vencimientos largos de seis a treinta años, para ser reemplazados por USD 400000 de bonos con vencimientos menores a tres años. Simultáneamente, se mantienen constantes los MBS reinvirtiéndolos para aumentar la duración y afectar los tipos a largo plazo. El objetivo de esta operación era dotar de liquidez al sistema sin aumentar la base monetaria, ya que, al financiarse mediante la venta de activos, no se hacía una inyección de capital (Morales, 2014, p. 26).

\footnotetext{
${ }^{2}$ En los Estados Unidos, existen instrumentos de inversión diferentes de una póliza de seguros emitidos por empresas, el Gobierno o instituciones, respaldados por hipotecas. Los GSE son títulos emitidos por empresas, apoyados por el Gobierno de los Estados Unidos, conocidos en inglés como governmentsponsored enterprises (GSE) y los MBS son valores hipotecarios conocidos en inglés como mortage backed security.
} 


\subsection{La tercera etapa (QE3)}

Para implementar entre noviembre de 2012 y diciembre de 2013, llamada Unlimited QE, la cual no fija una cantidad total por comprar, como se observa en las dos etapas anteriores; anuncia la FED que se comprarán USD 85 000 millones al mes, distribuidos USD 45 de treasuries y USD 40 de MBS. En esta etapa, se tiene como propósito comprar activos y aumentar la base monetaria para recuperar la economía disminuyendo la tasa de desempleo.
En mayo de 2013, la FED anuncia el fin de la política o tapering, mediante un recorte gradual de USD 10000 millones por mes finalizando en octubre de 2014 con un recorte de USD 15000 millones, una vez la economía de los Estados Unidos dé muestras de recuperación, con la reducción en la tasa de desempleo.

Tal como se menciona en Toro (2014), este anuncio de la terminación de la política trajo reacciones positivas y negativas, como el incremento de la tasa de interés de largo plazo y la consecuente volatilidad en los mercados financieros mundiales.

\section{Impacto de la política QE en los Estados Unidos}

D ara analizar el impacto de la política QE en la economía de los Estados Unidos, se han escogido algunos indicadores económicos representativos.

\subsection{Oferta monetaria}

Al anuncio de la política QE y su posterior implementación, el impacto en la economía de los Estados Unidos se vio con el incremento de la oferta monetaria, en busca del crecimiento del PIB. Se puede observar que esta variable se incrementó sustancialmente en las tres etapas de la flexibilización cuantitativa a partir de enero de 2009 y hasta finales de 2013 (Figura 5).

\subsection{Tasas de interés}

En efecto, la tasa de los bonos emitidos a diez años en los Estados Unidos, que se encontraba en $3.8 \%$ en 2009, llegó a ubicarse entre $1.4 \%$ y $1.7 \%$ en el tercer trimestre de 2012 y posteriormente en $2.7 \%$ a finales de 2014; una bajada en la tasa de interés por mayor confianza de los inversores sube el precio del activo y ayuda a mantener la tasa en términos normales (Morales, 2014, p. 31). 
Figura 5. Balance de la FED por periodos de QE-billones de dólares.

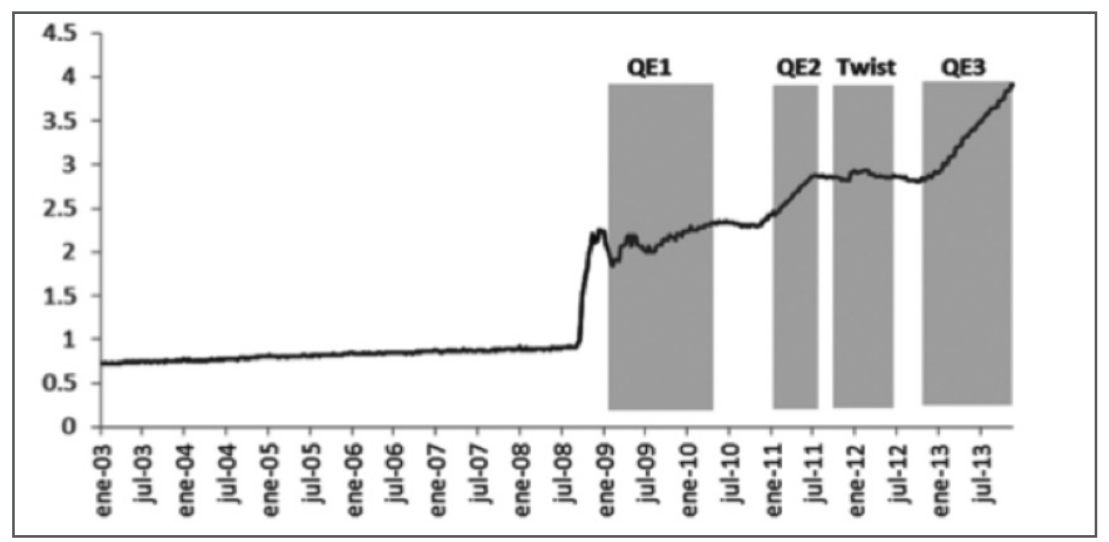

Fuente. Palomino (2013, p. 83).

\subsection{Tasa de desempleo}

Un indicador que permite ver la efectividad de la política monetaria es el índice NonFarm Payroll (NFP, nóminas no agrícolas). A continuación, se observa la tendencia positiva y creciente en las tres etapas del $\mathrm{QE}$, aunque no llega a la meta fijada por el Comité Federal de Mercado Abierto (FOMC, Federal Open Market Committee) (Palomino, 2013) (Figura 6).

Figura 6. NFP y QE.

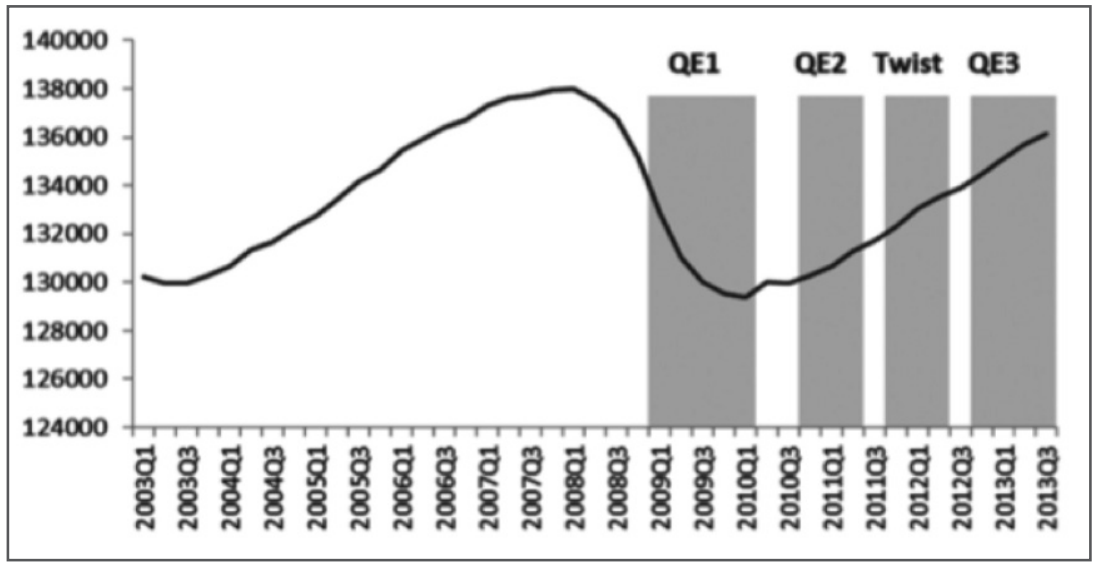

Fuente. Palomino (2013, p. 83).

Sin embargo, al correlacionar el balance de la FED con la tasa NFP, (Figura 7), se observa que en un periodo de tres años, entre enero de 2010 y enero de 2013, sí existe una fuerte correlación entre ambas variables con una tendencia al alza, que muestran un efecto positivo durante la implementación de las etapas 2 y 3 de la QE (Palomino, 2013). 
Figura 7. Balance de la FED y el NFP de enero 2004 a enero 2013.

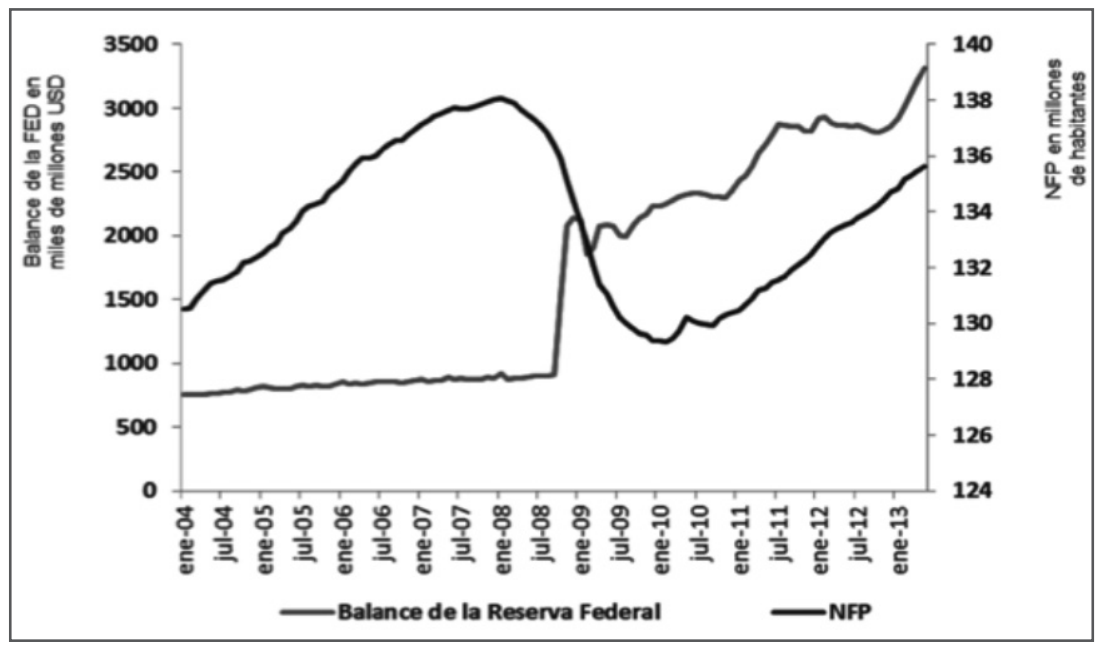

Fuente. Palomino (2013, p. 84).

\section{El impacto en la economía colombiana}

En Colombia, podemos ver el impacto de - la política de relajamiento cuantitativo de los Estados Unidos desde cuatro indicadores. El primero en la IED, el segundo sobre la tasa de cambio representativa del mercado (TRM), el tercero en el precio del crudo de Texas (WTI) y el cuarto el índice accionario COLCAP, en periodos que coinciden con la QE.

\subsection{La inversión extranjera directa}

El comportamiento de la IED en el país es un buen indicador de los efectos que ha recibido la economía colombiana durante el programa QE en los Estados Unidos. Un incremento del volumen de la inversión muestra el atractivo que tuvo para los inversores extranjeros la economía colombiana: en un principio, durante 2008 a 2010, hay una caída de IED cuando se inicia la recuperación de la economía de los Estados Unidos, en 2011 se reactiva la inversión hasta 2014 con un crecimiento importante que

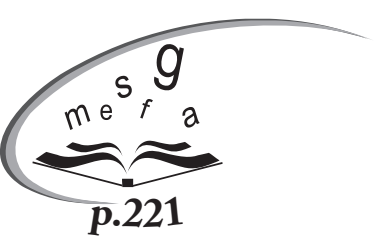
alcanza $153.9 \%$ en el periodo, al pasar de USD 6430 millones a USD 16325 millones. Este crecimiento está relacionado con el bajo riesgo, el fortalecimiento del peso frente al dólar y los altos ingresos por el precio del petróleo (Toro, 2015) (Tabla1 ). 
Tabla 1. Comportamiento trimestral de la IED 2008-2015 (millones de dólares)

\begin{tabular}{|l|l|l|l|l|l|l|l|l|l|l|l|l|l|l|l|l|}
\hline Trim & 2008 & $\%$ & 2009 & $\%$ & 2010 & $\%$ & 2011 & $\%$ & 2012 & $\%$ & 2013 & $\%$ & 2014 & $\%$ & 2015 & $\%$ \\
\hline 1 & 2718 & 26 & 2290 & 29 & 1206 & 19 & 3114 & 21 & 3533 & 23 & 3670 & 23 & 3815 & 23 & 3172 & 26 \\
\hline 2 & 2346 & 22 & 2790 & 35 & 1945 & 30 & 3104 & 29 & 4233 & 28 & 4015 & 25 & 4500 & 28 & 3914 & 32 \\
\hline 3 & 2598 & 25 & 1959 & 24 & 2349 & 37 & 3776 & 26 & 3327 & 22 & 4712 & 29 & 3724 & 23 & 2179 & 18 \\
\hline 4 & 2903 & 28 & 996 & 12 & 931 & 15 & 4654 & 32 & 3946 & 26 & 3812 & 24 & 3786 & 23 & 2844 & 23 \\
\hline Total & 10565 & & 8035 & -24 & 6430 & -20 & 14648 & 128 & 15039 & 3 & 16209 & 8 & 16325 & 1 & 12108 & -26 \\
\hline
\end{tabular}

Fuente. Cifras preliminares del Banco de la República, Subgerencia de Estudios Económicos. Cálculos del autor.

\section{2 Índice COLCAP}

Según la existencia de estudios de investigación (León y Trespalacios, 2015) utilizando la regla de MCO, en Parra (2016) se hace una regresión de las variables TRM y WTI, para ver el impacto en el índice accionario COLCAP entre 2010 y 2015, empleando el modelo de valoración de activos financieros (CAPM, capital asset pricing model), a partir de la siguiente ecuación:

$$
Y=\beta 1+\beta 2 \times 2 i+\beta 3 \times 3 i+\mu
$$

\subsubsection{Metodología para la estimación por el MCO}

Para observar el impacto de los indicadores macroeconómicos de interés, en los activos financieros de renta variable, desde su rendimiento, volatilidad y la relación con la $\mathrm{QE}$, se tomó el modelo de MCO utilizado en Parra (2016), dado que dicho método presenta propiedades estadísticas valiosas, que lo han convertido en uno de los más eficaces y habituales métodos en el análisis de regresión (Gujarati \& Porter, 2009) y ha sido utilizado con éxito en varias investigaciones como se menciona en León y Trespalacios, (2015), lo que da la confiabilidad del modelo de MCO para identificar la influencia de las variables y explicar el modelo CAPM, sin utilizar los modelos Garch de varianza condicional.
En el modelo, se aplica una regresión lineal para ver las combinaciones entre variables, aplicadas en el programa estadísticoecono-métrico Stata. De esta manera, se estiman los parámetros, se analiza su validez y se obtienen los resultados siguientes:

El modelo de MCO muestra lo siguiente:

Valor hoy $=2224.414-0.3638066$ trm + $0.0014543 w t i+e$

\section{$y=2224.414-0.3638066 \beta 1+$ $0.0014543 \beta 2+e$}

$\mathrm{Y}=$ valor hoy

cons $=$ constante

$\beta 1=$ TRM $\beta 2=$ WTI

$R$-squared = El coeficiente de determinación es suficientemente alto, es muy cercano a 1 (0.8612); por tanto, expresa que la calidad del modelo en este caso es óptima.

$\mathrm{P}>[\mathrm{T}]=p$-value o valor $p$ es una medida de significancia estadística que está entre 0 y 1; para este model, se toman como nuestras hipótesis =

Hipótesis nula $(\mathrm{Ho})=$ los índices de petróleo WTI y TRM no explican el índice de COLCAP $p>0.05$ 
Hipótesis alterna $(\mathrm{Ha})=$ Los índices de petróleo WTI y TRM explican el índice de COLCAP $\mathrm{p}<0.05$

Sensibilidad de las variables independientes:

La elasticidad de la TRM es negativa $(-0.3638066)$

La elasticidad del WTI es positiva (0.0014543)

Como se puede leer, la TRM y WTI tienen un $p$-value $<0.05$, por tanto, se acepta la hipótesis alterna; es decir, ambas variables explican el índice de COLCAP, de tal forma que un aumento de un dólar en $\beta 1$ hace que $Y$ disminuya en (0.3638066) dólares y para B2 aumentaría en 0.0014543 dólares, en concordancia con las elasticidades de la TRM y del WTI.

Por ejemplo, si la TRM sube a 3500 y el WTI baja a 3500, el índice COLCAP será de

\section{$y=2224.414-0.3638066(3500)+0.001$ $4543(35000)+e$ $y=1001.99$}

En el estudio realizado, se concluye que, a medida que sube la TRM, el índice COLCAP disminuye, por tanto, existe una relación indirecta:

\section{$y=2224.414-0.3638066 \beta 1$}

Se pudo observar que los índices COLCAP y WTI son directos, es decir que, a medida que el WTI crece, el COLCAP también lo hace:

$$
y=2224.414+0.0014543 \beta 2
$$

Se muestra una caída del índice COLCAP en 2011, (Fifgura 8), periodo que coincide con la segunda etapa de la QE en los Estados Unidos; durante 2012 se presenta un incremento acentuado del índice durante el año. Estos dos resultados son consistentes con las evidencias encontradas en Leyva, Gómez, Valencia y Villamizar (2016). En efecto, hay un impacto de la política QE en el índice accionario COLCAP negativo en la segunda etapa y positivo en la tercera. En 2013, se observa un descenso pronunciado del índice debido a los anuncios de intervención de Interbolsa por la Superintendencia Financiera de Colombia y la posterior quiebra de la entidad (Gómez y Melo, 2013).

En 2014, se observa una recuperación del índice COLCAP que llega al tercer trimestre, a partir de esta fecha comienza un descenso pronunciado que alcanzó su nivel más bajo a finales de 2015. El comportamiento negativo del indicador accionario desde finales de 2014 se debe, especialmente, a la salida de capitales hacia el exterior.

Los anteriores resultados indican que durante el periodo de aplicación de la política de flexibilización monetaria en los Estados Unidos, el ingreso de capitales extranjeros impactó el índice COLCAP positivamente durante las etapas uno y tres y negativamente en la segunda. Al presentarse los anuncios de política monetaria en 2014 y hasta diciembre de 2015, los resultados son adversos por la salida de capitales hacia los Estados Unidos, el endurecimiento de la política monetaria en ese país y el fortalecimiento del dólar que impactó la divisa colombiana y ayudó a su devaluación; en segundo término, se puede mencionar la reducción del precio del petróleo. 
Figura 8. Comportamiento del índice COLC.AP (junio 2011-junio 2015).

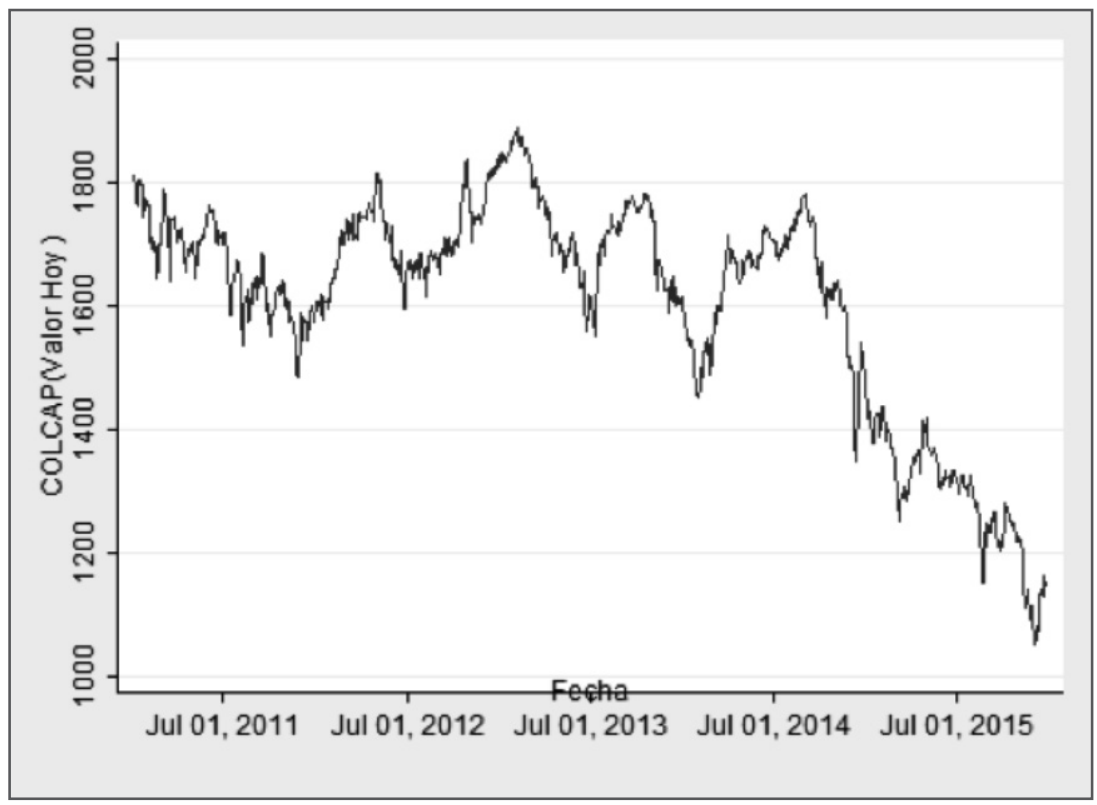

Fuente. Parra, (2016).

\subsection{Otras reflexiones sobre los indicadores en Colombia}

Siguiendo a Toro (2014), la implementación de la QE en los Estados Unidos trajo una

secuencia de hechos en economías emergentes: aumento de las entradas de capital de portafolio; reducción de la percepción del riesgo; apreciación de las monedas; disminución de las tasas de interés de los activos financieros locales; estímulo a la dinámica del crédito y alza en los precios de los activos reales y financieros.
En Colombia, los cambios en algunos de los indicadores tuvieron reacción inmediata, entre ellos:

- El impacto en la IED de portafolio, que pasó de negativo en 2010 a superar 1.5 $\%$ del PIB.

- La creciente participación del capital extranjero en el mercado de deuda pública que contribuyó al incremento del precio de los TES.

- Con el anuncio de la reducción de compras de activos en los Estados Unidos, en diciembre de 2014, en Colombia la tasa de cambio se mantuvo estable, las acciones se valorizaron y el EMBI emerging markets bonds index - o riesgo país se redujo en $1.14 \%$. 


\section{El impacto de quantitative easing en las bolsas de valores del mundo}

Entendido que la QR es una política -monetaria que busca reactivar la economía cuando los lineamientos tradicionales no tienen un efecto deseado, el instrumento utilizado aumenta la BM con las compras masivas que hace el Banco Central de la deuda corporativa o del Gobierno, que, en última instancia, incrementa el dinero en circulación para que aumente el crédito y, en consecuencia, se incremente la inversión activando la economía.

Según el estudio realizado por Costero (2013), no hubo aumento en el ingreso, ni en la inflación ni en el empleo. Sin embargo, sí se presenta una reacción en las bolsas de valores del mundo (Figura 9), en el cual se destaca el comportamiento de los principales índices accionarios en cada una de las etapas del programa QE, como el DAX40, el S\&P500, el NINKEI, el CAC40, el IBEX35 y el ASE.

Los valores de los índices aumentan superando los máximos en las tres etapas del programa, con excepción de ASE de Grecia. Y estos efectos positivos son reacciones lógicas entre los inversionistas, debido a la teoría de las expectativas (Bodie, 2008, p. 240), dado que la política expansiva incrementa la liquidez, permite el incremento de los precios de los activos de renta variable y se reduce el rendimiento de la renta fija. No hay que olvidar que los inversionistas buscan mercados financieros donde puedan incrementar el rendimiento de sus portafolios.

La reacción también se presenta en las divisas y en los bonos. Según declaraciones del ministro de Finanzas de Brasil, se presentó una guerra de divisas entre países que los llevó a decidir el futuro de la economía con una política monetaria expansiva que colaborara con la reducción de los valores de sus monedas y demanda alta en los bonos, cuyas subastas fueron por la baja de las tasas (Costero, 2014).

\subsection{Retorno de los indicadores accionarios de los países emergentes}

Siguiendo a Leyva et al. (2016), la política de la QE tuvo un impacto significativo en el retorno de los índices accionarios de las bolsas de valores de países emergentes ubicados en América, Europa, África y Asia. El resultado muestra que el QE1 afectó en $7.314 \%$ el retorno de los índices accionarios, el QE2 afectó en -2.988\% el retorno de los índices accionarios y el QE3 afectó en 2.290 $\%$ el retorno de los índices accionarios de la muestra de países. Hay un impacto positivo en el retorno de los índices accionarios en la QE1 y QE3 y negativo en la QE2; esta razón negativa se explica por el cambio de esquema de financiación de las compras de activos de largo plazo, lo cual llevó a la modificación del portafolio de los inversores y su curva de rendimientos.

Resaltan estos investigadores que el efecto agregado de todo el programa de compras de activos es positivo en todas las regiones aplicadas en el estudio y en el total de la muestra. Concretamente, el efecto sobre el retorno de los índices del total de la muestra es un incremento de $6.616 \%$, lo cual permite afirmar que tuvo un impacto positivo en los índices con la aplicación de la $\mathrm{QE}$, por el flujo de capitales que llegó a los mercados financieros externos (Figura 9). 
Figura 9. Efecto de la QE en las bolsas del mundo.

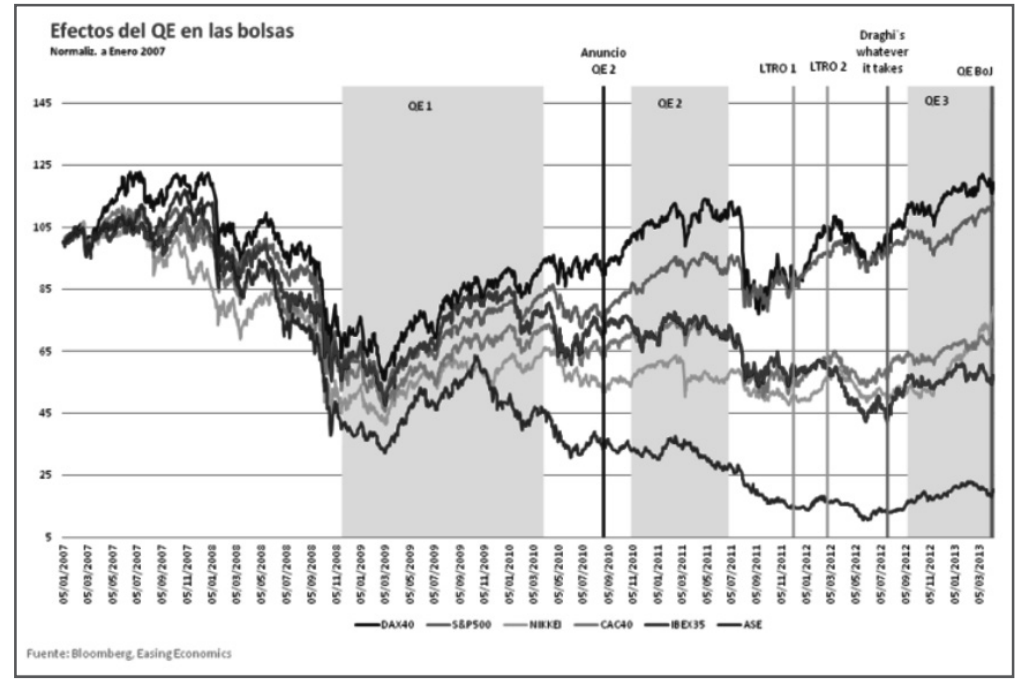

Fuente. Costero, (2013).

\section{Ventajas y desventajas en la aplicación de una flexibilización cuantitativa}

Toda política, económica, monetaria, administrativa, tiene sus ventajas y desventajas. En este caso, la QE tiene efectos que redundan en beneficio del conjunto de la economía y que permitirían reanimarla y también desventajas que es importante tener en cuenta para ajustes o posibles anticipaciones.

Partiendo del análisis que hace el economista Juan Ramón Rallo (2015), se han tomado los principales aspectos positivos y negativos que puede traer una política de flexibilización monetaria.

\subsection{Ventajas o aspectos positivos}

Estas son las ventajas o aspectos positivos:

- Una flexibilización monetaria contribuye a reducir los tipos de interés debido al aumento de la demanda de los títulos de deuda por el banco central, lo cual constituye un estímulo para las entidades cuyos títulos de deuda adquiere el banco central; los menores tipos de interés reducen el costo de financiación.

- La posición financiera de los bancos comerciales mejora, ya que sus inversiones no están comprometidas en los tradicionales créditos de largo plazo, sino que son sustituidas por la creación del efectivo del Banco Central.

- Los menores tipos de interés y la inyección de dinero a los bancos privados incrementa la oferta y el otorgamiento de nuevos créditos al sector productivo. El balance de los bancos se hace más sólido y puede disponer de mayor capacidad para otorgar créditos. 
- Las bajas tasas de interés de la deuda pública son un incentivo para que el sector bancario mire nuevas opciones para colocar su dinero, debido a que la compra de deuda ya no es atractiva al anular-se la rentabilidad por la operación de flexibilización monetaria del Banco Central.

- Como consecuencia, habrá una mayor cantidad de bancos deseosos de prestar, lo cual repercute en la caída de los tipos de interés del crédito atrayendo el deseo de endeudamiento del sector empresarial y las familias.

- Uno de los aspectos más relevantes es el aumento del crédito al sector privado que va a permitir incrementar el consumo y la inversión en sectores productivos. Por principio económico, el mayor gasto privado hará reaccionar positivamente la economía y evitar la deflación por el incremento de precios.

- Las finanzas internacionales también se ven mejoradas proporcionalmente por el gasto al crédito que se filtra al exterior. Es claro que habrá que vender la divisa del país que aplica la flexibilización cuantitativa y comprar de las demás divisas que permite la depreciación de su moneda y la apreciación de las externas.

- Quizá lo más importante es que la mayor disponibilidad de crédito a bajo costo, el mayor gasto interno, las subidas de precios, la depreciación de la moneda y las expectativas de que estas condiciones de política monetaria relajada se mantengan por un largo plazo se constituyan en una política macroeconómica que incremente el crecimiento del PIB, con un bajo índice de desempleo y una inflación estable y cómoda o reducida.

\subsection{Desventajas y contraindicaciones}

Las siguientes son las principales desventajas y contraindicaciones:

- El primer efecto es que el Banco Central pierde margen de maniobra en el momento de gestionar su moneda. La QE esteriliza los instrumentos tradicionales con los que cuenta el Banco Central para administrar su provisión de financiación al sistema financiero. En este sentido, la estrategia de ingreso en la política de flexibilización cuantitativa está muy clara, pero la estrategia para salir de esta política hasta el momento no parece fácil.

- Un segundo efecto es que los bajos tipos de interés no tienen por qué estimular un nuevo ciclo de endeudamiento para relanzar la economía al crecimiento, salvo en el sector público, cuyos costos se trasladan a los contribuyentes futuros. Pero, en el caso de las empresas privadas y el consumidor común, facilitarles el endeudamiento no los llevará a redoblar sus pasivos, lo cual haría que los bancos asuman un costo de oportunidad con sus recursos en tesorería. Lo anterior se puede interpretar como la reducción de tipos de interés de la flexibilización cuantitativa que consolidará un contexto económico de alto endeudamiento y de activos sobrevalorados, lejos de facilitar el reajuste económico.

- Con relación a los efectos en el sistema bancario, las menores tasas de interés estimulan los nuevos créditos, lo cual puede llevar a las entidades bancarias a que asuman mayores riesgos en el futuro. Más deuda y más riesgo, más fragilidad financiera a largo plazo que fue lo que 
condujo a la crisis de 2008 (Stiglitz, 2010), por lo que se debe exigir una mayor regulación o control.

- Un efecto impactante para los actuales deudores bancarios es que las tasas bajas estimulan el crédito con costo cero o muy reducido y en muchos casos han asumido operaciones anteriores con tasas más altas que muestran una deuda con desventaja competitiva. De esta forma, se financian proyectos de inversión inseguros y se concede una ventaja a los más inseguros.

\section{Conclusiones}

U na política monetaria que pretenda la expansión del PIB con los instrumentos convencionales de bonos y crédito no es buen sustituto de los efectos positivos que genera la política monetaria expansionista con la QE. Como lo expresan Bernanke y Blinder (2012), se requiere instrumentos diferentes de los tradicionales para incentivar el crecimiento económico; por esto, la compra de bonos del Gobierno y los bonos corporativos aumentan la oferta monetaria y dan mayor liquidez a la economía.

La política de expansión monetaria para incentivar el crecimiento de la economía de los Estados Unidos a través de la QE, después de la crisis de 2007-2008, ha tenido efectos positivos y negativos. La base monetaria se ha incrementado y permitido liquidez en la economía, como consecuencia de la QE-1 que fue notable; rol similar cumplió la QE-2; e igualmente lo hizo la QE-3. Sin embargo, los instrumentos de política monetaria implementados no han sido suficientes para llevar la economía de loa Estados Unidos a su pronta recuperación, a pesar de contar con la reducción de las tasas de crédito, incremento de la BM y compra de bonos del Gobierno y bonos corporativos.

En el caso colombiano, la economía se ha visto beneficiada entre 2008 y 2014 por el ingreso de capitales foráneos que beneficiaron la IED y los precios de acciones reflejados en el índice COLCAP. La TRM se mantiene baja y estable hasta el año 2014 por el ingreso de IED y las regalías por exportaciones que aumentan la oferta de divisas lo que genera una revaluación; posteriormente se inicia una devaluación a partir de la finalización del programa QE y los anuncios de la FED de endurecer la política monetaria, lo cual se hace real a finales de 2015.

El mayor beneficio se ve en los mercados de capitales, ya que las bolsas de valores del mundo y, en especial, la colombiana recibieron un impacto positivo durante las etapas de implementación de la $\mathrm{QE}$, con excepción de la segunda, toda vez que la política QE era expansionista, razón por la cual se frenaron las expectativas de los inversionistas sobre el futuro del programa.

Desde este punto de vista, los resultados encontrados en Costero (2013), Palomino (2013) y Leyva et al. (2016) coinciden en que la política de flexibilización monetaria tuvo un impacto positivo de significancia en la oferta monetaria, hay un efecto notorio con el incremento del empleo aunque no como se esperaba y una marcada influencia en las bolsas de valores de las economías emergentes como la colombiana. 


\section{Referencias}

BBC Mundo. (2009). La crisis financiera: una cronología -agosto 2007 a septiembre 2008. Recuperado de http://news.bbc. co.uk/hi/spanish/ specials/2008/crisis_financiera/ newsid_7629000/7629122.stm

Barcelata Chávez H. (2010). La crisisfinanciera en Estados Unidos. Contribuciones a la Economía. Recuperado de http://www. eumed.net/ce/2010a/hbc.htm

Barro, R., Grilli, V. y Febrero, R. (2004). Macroeconomía: teoría y política (4. ed.). México: McGraw-Hill.

Bernanke, B. S. y Blinder, A. S. (2012). Credit, money and aggregate demand. American Economic Review, 78(2), 435-439.

Bodie, Z. (2008). Investments(7. ed.). Nueva York, NY: McGraw-Hill.

Castañeda, J. (2012). Porqué se utilizan operaciones de expansión cuantitativa QE? En Oroyfinanzas.com. Recuperado de https://www.oroyfinanzas.com/2012/11/ por-que-utilizanoperaciones-expansion-cuantitativa-qequantitative-easing/

Costero, J. (2013). Easing the Quantitative (II): Efectos de la QE-De-sarrollados. En Easingeconomics.com. Recuperado de https://easingeconomics.wordpress. com/2013/05/13/easing-the-quantitativeii-efectos-del-qe/

Costero, J. (2014). ¿Ha tenido éxito el Quantitative Easing? En Oroyfinanzas. com. Recuperado de https://www. oroyfinanzas.com/2014/11/ha-tenidoexito-quantitative-easing/

Gómez, J. E. y Melo, L. F. (2013). Efecto de ángeles caídos en el mercado accionario colombiano: estudio de eventos en el caso Interbolsa. Borradores de Economía, 779. Recuperado de http://www.banrep.gov. co/es/borrador-779

Gujarati, D., \& Porter, D. (2009). Econometría, Quinta edición. México: McGraw-Hill.

León Cristancho, S. J. y Trespalacios Carrasquilla, A. (2015). Factores macroeconómicos que influyen en la volatilidad del índice accionario COLCAP. Recuperado de https://studylib.es/doc/7676095/ factores-macroecon \%C3 \%B3micos-queinfluyen-en-la-volatilidad-d...

Leyva, B., Gómez, J. E., Valencia, O. M. y Villamizar, M. (2016). Efectos del quantitative easingsobre los retornos accionarios. Borradores de Economía, 929. Recuperado de http://www.banrep. gov.co/en/node/40738

Morales Solá, P. (2014). La eficacia de las políticas monetarias de quantitative easing llevadas a cabo por la Reserva Federal de los Estados Unidos y por el banco Central Europeo (Tesis de grado, Universidad Pontificia Comillas, Madrid, España).

Palomino Selem, C. (2011). Crisis financiera internacional: enfoque en instrumentos y riesgos. Pensamiento Crítico, 16, 69-77. 
Palomino Selem, C. (2013). Efectos de la política monetaria de flexibilización cuantitativa en Estados Unidos de América sobre el empleo. Pensamiento crítico, 18(2), 7794.

Parodi Trece, C. (2013). Crisis económica mundial e impactos sobre el Perú. En Los Andes. Recuperado de http://www.losandes.com.pe/Economia/20130825/74217.html

Parra Barrios, A. (2016). Tasa representativa del mercado y WTI: determinantes en las variaciones del índice COLCAP entre 2010 y 2015. Revista Faccea, 6(2), 146-153.
Rallo J. R. (2015). ¿Qué es y qué efectos tiene una flexibilización cuantitativa? En Elcato.org. Recuperado de https://www. elcato.org/que-es-y-que-efectos-tiene-unaflexibilizacion-cuantitativa

Stiglitz, J. E. (2010). La caída libre: el libre mercado y el hundimiento de la economía mundial. Madrid: Taurus.

Toro, J. (2014). El relajamiento cuantitativo en los Estados Unidos y algunos efectos en Colombia. Reportes del Emisor, 176. Recuperado de http://www.banrep.gov. co/es/emisor-176

Zablotsky., E. (2011). Modelo IS-LM. Recuperado de https://goo.gl/tPkY78 